\title{
Standardization of in vitro testing for cardiovascular implants in the era of European Medical Device Regulation
}

\author{
Wolfram Schmidt, University Medicine Rostock, Institute for Biomedical Engineering, Rostock, Germany, \\ wolfram.schmidt@uni-rostock.de \\ Christoph Brandt-Wunderlich, Michael Stiehm, Sebastian Kaule, Stefan Siewert, Andrea Bock, Institute for Im- \\ plantTechnology and Biomaterials IIB e.V., Rostock, Germany \\ Klaus-Peter Schmitz, Institute for ImplantTechnology and Biomaterials IIB e.V., Rostock, Germany, and University \\ Medicine Rostock, Institute for Biomedical Engineering, Rostock, Germany \\ Anja Kurzhals, Niels Grabow, University Medicine Rostock, Institute for Biomedical Engineering, Rostock, Germany
}

\section{Introduction}

Technical standards for medical devices represent a common sense of the current state of technology for use in product development, approval and their safe and efficient use. Cardiovascular implants as class III medical products require a specific responsibility from manufacturers and regulatory authorities. The complex process of standards development is driven by the need to adapt the fast development of medical and technical science.

\section{Methods}

The study is considering normative and informative guidance from standardization under the new requirements of the European Medical Device Regulation (MDR). A summary of international standards is analyzed with respect to their use for cardiovascular implants such as drug-eluting stents and artificial heart valves. The need for new test methods is derived presenting technical solutions as examples.

\section{Results}

A list of standards from the International Organization for Standardization (ISO) and the American Society for Testing and Materials (ASTM International) was identified for requirements on arterial stents, stent grafts, bioresorbable and drug-eluting stents as well as implants for transcatheter aortic valve replacement (TAVR). Additional guidance is given by national authorities such as from the U.S. Food and Drug Administration (FDA). New issues cover among others the characterization of coating integrity and particulate evaluation as well as the assessment of the haemolytic and thrombogenic potential of implants for TAVR.

MDR requires technical expertise and capacity at Notified Bodies. The Technical Documentation and the clearly accented responsibility of manufacturers requires high-sophisticated test procedures also for independent laboratories.

\section{Conclusion}

The testing of cardiovascular implants is highly regulated but requires permanent development to protect the high quality level. The interest in standardization from industry, test laboratories and authorities is high, but more input from medical experts would further improve the value of standardization and its relevance for safe and even more effective implants. 


\section{Medical Device Regulation and current challenges for the implemen- tation of new technologies}

Sebastian Kaule, Institute for Implant Technology and Biomaterials e.V., Rostock-Warnemünde, Germany, sebastian.kaule@uni-rostock.de;

Ernst Klar MD, FACS, Department of Surgery, University Medical Center Rostock, Germany,

Thomas Lenarz MD, Department of Otolaryngology, Hannover Medical University, Germany, Matthias Leuchter, Department of Surgery, University Medical Center Rostock, Germany,

Ariane Dierke, Institute for Implant Technology and Biomaterials e.V., Rostock-Warnemünde, Germany,

Marek Zygmunt MD, Clinic and Polyclinic for Gynaecology and Obstetrics, University Medical Center Greifswald, Germany

Wolfram Schmidt, Institute for Biomedical Engineering, University Medical Center Rostock, Germany

Niels Grabow, Institute for Biomedical Engineering, University Medical Center Rostock, Germany

Andrea Bock, Institute for Implant Technology and Biomaterials e.V., Rostock-Warnemünde, Germany,

Klaus-Peter Schmitz, Institute for Implant Technology and Biomaterials e.V., Rostock-Warnemünde, Germany, | Institute for Biomedical Engineering, University Medical Center Rostock, Germany

Stefan Siewert, Institute for Implant Technology and Biomaterials e.V., Rostock-Warnemünde, Germany,

Michael Stiehm, Institute for Implant Technology and Biomaterials e.V., Rostock-Warnemünde, Germany,

The European Parliament and the European Council on May 26, 2017 decided to introduce the new regulatory framework for medical devices. The transitional period of the so called Medical Device Regulation (EU 2017/745, MDR) should end on May 26, 2020. Currently, about one month before this date, the European Commission is working on a postponement for one year to relieve pressure from all stakeholders allowing them to fully focus on priorities related to the coronavirus crisis.

Most likely from May 26, 2021 manufacturers must present a CE-certificate according to the new MDR requirements not only for novel medical devices, but also for approved medical devices which are already on the market. The MDR will significantly complicate the process of bringing medical devices into market due to the increased requirements for the CE-certification process, particularly concerning increased documentation effort.

This involves a risk for the translation of innovative products due to an overload of the overall system and might lead to shrinkage of the product range of existing products. Thankfully, adaption of transitional periods, special regulations, extensions of deadlines and most probably postponement of the MDR application date will ensure that there will be no gaps in medical supply that endanger patients.

Establishing and keeping the relevant regulatory expertise up to date, devoting the necessary financial, time and human resources that is the biggest immediate challenge the medium-sized medical technology sector is facing in the near future.

The conference contribution in fall 2020 will summarize the main aspects of the current state of MDR implementation with respect to regulatory novelties, the current legal basis in Germany, transition periods and changes in the requirements of CE-certification relevant technical documentation.

Furthermore, challenges for existing and innovative medical devices will be discussed briefly using a new stent based therapy of the proximal Fallopian tubal stenosis and transcatheter aortic valve implantation as examples. 


\section{First detected cases of service shortages regarding medicinal product using patients in pandemic isolation}

Stefanie Rödig, MainSport, Department Research and Ethics, Seligenstadt, Germany, stefanie.roedig@mainsport.info; Stephan J. Schließmann, Integrative and Experimental Exercise Science \& Training, Institute of Sport Science, University of Würzburg, Würzburg, Germany; MainSport, Department Research and Ethics, Seligenstadt, Germany, stephan.schliessmann@uni-wuerzburg.de

\section{Introduction}

In response to the outbreak of SARS-CoV-2, the Federal Government of Germany prescribed guidelines for limitation of social contacts. These policies resulted in social, economic and systemic restrictions.

Several countries concerned before reported on device and equipment shortages of mechanical ventilators, disinfectant products, and face masks in clinical settings.

The aim of our study was the contemporary investigation of the potentially existence of medicinal product problems in a home setting of chronically ill people during the time of distancy and isolation.

\section{Methods}

210 adult participants for this study were recruited through posts in online groups. Individuals provided feedback via an anonymous questionnaire.

Any kind of medicinal product following the German Medicinal Product Act (MPG) used by patients were included in this study to investigate consultation difficulties and service shortages regarding the medicinal products. To identify only problems of chronically ill people, we included answers of patients supplied with oxygen at home and analyzed the statements in a qualitative examination.

\section{Results}

Pandemic related difficulties in medicinal product related problem solving have been identified in the first weeks of social distancing, isolation and quarantine.

Device problems, not solved by users themselves, encountered systemic difficulties in isolation. Problems in replacing medicinal products, hygienic deficiencies and lack of devices have been reported.

\section{Conclusion}

We detected first cases of service providing bottlenecks in the field of medicinal products during the first weeks of pandemic restrictions in Germany. The results of our study diclose that more detailed information and further comprehensive studies in the field of medicinal products are reqired to find out more about systemic deficiencies for patients' safety in isolation and quarantine. 